\title{
Vertical profiles of giant particles close above the sea surface
}

\author{
By G. DE LEEUW, Physics and Electronics Laboratory TNO, P.O. Box 96864, 2509 JG The Hague, The \\ Netherlands
}

(Manuscript received August 20; in final form December 3, 1985)

\begin{abstract}
Size distributions of giant sea-spray particles $(10-100 \mu \mathrm{m}$ diameter) were measured in the North Atlantic with an impaction method, at heights of $0.2,0.5,1,2,4,6$ and $11 \mathrm{~m}$ above instantaneous sea level. The vertical distributions of the particle concentrations show as characteristic features a minimum at $0.5 \mathrm{~m}$ at low winds, and at higher winds a maximum at $1-2$ $\mathrm{m}$. The windspeed dependence of particle concentrations is a function of both particle diameter and height above sea level. The results are explained by a qualitative model based on literature data on the production of sea-spray particles and on turbulent wind structure over water waves.
\end{abstract}

\section{Introduction}

The vertical structure of particle size distributions in the marine atmosphere is of importance for direct practical applications (e.g., performance predictions of electro-optical equipment, deposition studies (Fairall and Larsen, 1984; Slinn and Slinn, 1980), production estimates of marine aerosol (Fairall et al., 1983)), as well as for more theoretical purposes (e.g., modelling of the marine atmosphere for optical and meteorological purposes).

Of particular interest for studies on particle transport phenomena is the near-surface layer $(\mathrm{Wu}$, 1979). Freshly produced particles are ejected to heights limited to less than $20 \mathrm{~cm}$ (Blanchard and Woodcock, 1957; Blanchard, 1983), so external upward forces are required for transport from the surface into the mixing layer. Various approaches are applied to estimate production rates of marine aerosol (cf. Fairall et al., 1983; Monahan, 1985) or deposition of particles on natural waters (cf. Slinn, 1983 for a review). It has been recognized, however, that for a better description of particle transport, measurements of the vertical distributions of particle concentrations to close above the sea surface are required (Wu, 1979; Fairall and Larsen, 1984).
In view of the interest in vertical distributions of particle concentrations, little experimental work has been done and most measurements were made at altitudes of about $10 \mathrm{~m}$ and higher (see below, however). From compilation of the available data, particle concentrations are expected to be highest near the surface and to decrease toward higher altitudes (Blanchard and Woodcock, 1980; Blanchard et al., 1984). This picture is confirmed by the model of Toba (1965) and by the few data which were reported on the height dependence of particle concentrations close above the sea surface. Size distributions measured at heights between 1 and $13 \mathrm{~m}$ were published by Chaen (1973), who concluded that on the average the vertical distributions of the number concentrations approached a power law dependence. Preobrazhenskii (1973) concluded from droplet spectra measured between 1-2 and $7 \mathrm{~m}$, that the particle volume decreased exponentially with height. To our knowledge, no other field data are available on the vertical structure of aerosol concentrations close above the sea surface. Size distributions of very large particles at very low height $(0.13 \mathrm{~m})$ were measured by Monahan (1968).

Furthermore, some laboratory studies concerning the height dependence of particle size distribution close above the water surface were 
reported (cf. Wu (1979) for a review). Comprehensive experiments were recently made by Koga and Toba (1981) and Koga (1984), who determined both fluxes and trajectories of particles in a windtunnel with different techniques. For particles in the submm range, vertical profiles were constructed which extend into the wavetroughs (Koga and Toba, 1981). The results reveal a maximum in the particle concentrations which was not observed in the field data mentioned above, probably because in the latter the sampling heights were not close enough to the sea surface.

In this paper, size distributions of giant sea-spray particles are presented, measured in the North Atlantic from very close to the instantaneous sea surface $(0.2 \mathrm{~m})$ up to $11 \mathrm{~m}$. Particle spectra were determined with a Rotorod impaction sampler as described in Section 2. Particle profiles were constructed from these data which confirm the existence of a maximum as observed by Toba and Koga (1981) from laboratory experiments. However, the shapes of the profiles are strongly dependent on windspeed. The results are explained by a qualitative model based on previously published observations of particle production (e.g., Blanchard and Woodcock, 1957) and on studies of turbulent wind structure over water waves (Hsu et al., 1981; Konishi, 1981; Banner and Melville, 1976; Anisimova et al., 1976).

The measurements described in this paper were performed in May/June 1983 in the North Atlantic at station Lima $\left(57^{\circ} \mathrm{N}, 20^{\circ} \mathrm{W}\right)$, aboard the Netherlands weathership MS Cumulus, as part of a programme to study the optical and infra-red properties of the marine atmosphere.

\section{Experiments}

Particles in the diameter range from 10-100 $\mu \mathrm{m}$ were sampled using a Rotorod inertial impactor (manufactured by Ted Brown Ass.), consisting of two rods, $8.3 \mathrm{~cm}$ apart, mounted on a motor which rotates at a speed of $2400 \mathrm{rpm}$. The linear velocity of the rods is $10 \mathrm{~m} / \mathrm{s}$. For our purpose, stainless steel rods were used of $5 \mathrm{~cm}$ long and $1.6 \mathrm{~mm}$ in diameter, polished mirror flat at one side over a width of $1.5 \mathrm{~mm}$. Silicone was sprayed over the polished surface as a sticky coating to hold the particles, which due to their own inertia are sampled by the rotating rods. In order to prevent collection of particles by convection or diffusion before the start of the sampling, a retracting collector head was used, modified for our stainless steel rods.

The exposed rods were examined through a microscope focussed on the smallest particles. Five microscope images, chosen at random over the area of each of the two rods forming a sample, were photographed. The ten photographs were later digitized and stored in the memory of an image processing system, to determine the particle size distribution automatically. For each size distribution, a total number of about 1000 particles was counted. The diameter range from $10-100 \mu \mathrm{m}$ was logarithmically divided into 8 intervals, with median diameters of $11,15,20,28,37,48,64$ and 85 $\mu \mathrm{m}$. The particle size distributions $\mathrm{d} N / \mathrm{d} D\left[\mu \mathrm{m}^{-1}\right.$ $\mathrm{cm}^{-3}$ ] were obtained by dividing the number of particles in each interval through the interval width and the sampling volume.

The Rotorod is an active impaction sampler, as opposed to passive methods such as coated glass slides etc. as used in the measurements quoted in the introduction (Chaen, 1973; Preobazhenskii, 1973; Monahan, 1968; Koga and Toba, 1981). With the latter, horizontal particle fluxes were measured, which were converted to particle concentrations by using some assumptions, $c f$. Koga and Toba (1981). The Rotorod collects particles by taking advantage of their inertia as well, but the air sampled is not only refreshed by natural convection (wind), but also by aspiration due to the rotation of the rods.

Particles larger than about $10 \mu \mathrm{m}$ are efficiently $(>90 \%)$ collected by the impaction rods used in our experiments, as verified both from theory (Emonds, 1972) and practice (Vrins and Hofschreuder (1983) and references therein). Sampling efficiency is only slightly influenced by windspeed (Vrins and Hofschreuder, 1983). However, the wet aerosol sampled in marine conditions might behave differently from the monodisperse organic droplets or the glass beads used for calibration, while errors also might be introduced by evaporation during transport and processing of the samples. Therefore, a number of Rotorod samples was closely examined. It appeared that after the evaporation of water vapour in the relatively dry laboratory environment, crystals were left which often were deeply embedded in the silicone coating. These crystals could only be 


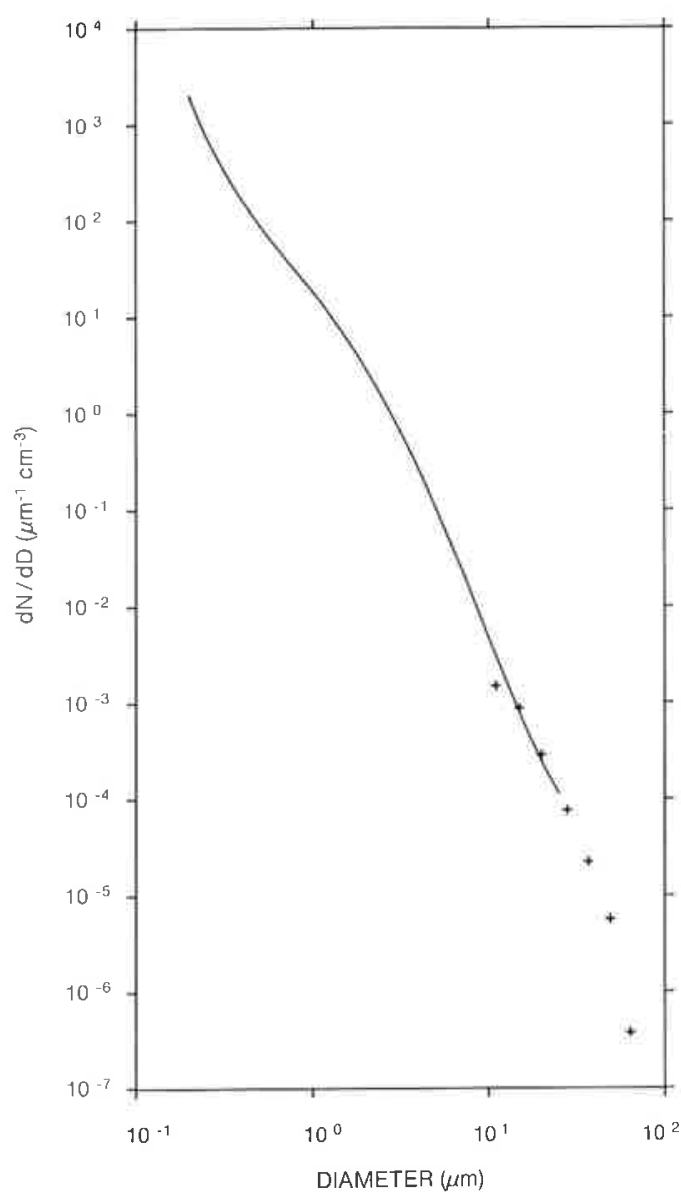

Fig. 1. Particle size distributions measured simultaneously at $11 \mathrm{~m}$ above sea level with the Rotorod $(+)$ and with optical particle counters (polynomial fit to the data (Van de Ven et al., 1980), represented by the solid line). Particle diameters are for ambient relative humidity $(68 \%)$.

observed by their reflection and scattering when the microscope was focussed under the toplayer of the silicone coating. The dimensions of the craters in which the crystals were left, however, correspond closely to the diameters the particles had in the atmosphere, as confirmed by studies on sampling of mono-disperse aerosol with $\mathrm{MgO}$ coatings (F. Oeseburg, 1984, private communication). Hence, when the microscope is properly focussed, the particle size distributions obtained from Rotorod samples are representative for the atmospheric aerosol (see also de Leeuw, 1986). This is con- firmed by comparison of the Rotorod data with spectra measured simultaneously and at short distance with PMS optical particle counters. As shown in the example of Fig. 1, the particle concentrations measured with the two instruments are in good agreement in the overlapping size range.

The Rotorod impaction sampler was used to determine particle size distributions at heights of $0.2,0.5,1,2,4,6$ and $11 \mathrm{~m}$. The latter three are the average heights of the decks of MS Cumulus, where the samples were collected at the windward side, at short distance outside the rail. The measurements at the four lower heights were made with the Rotorod mounted on a simple wave follower, consisting of a toroidal buoy fastened to gimbals on a tube, which could slide freely up and down a taut rope. Due to the gimballed attachment the buoy follows the slopes of the waves, as well as water level. The Rotorod was mounted on a $40 \mathrm{~cm}$ extension rod, to reduce the influence of the buoy on the measured particle size distributions. The variation in the sampling height due to the wave motions is estimated to be about $10 \mathrm{~cm}$. In very rough seas, sampling at $0.2 \mathrm{~m}$ was skipped to avoid the risk of drowning the sampler. To reduce the influence of the ship, the measurements with the wave follower were performed on the windward side at about $6 \mathrm{~m}$ from the hull, while the ship drifted in the wind and waves. Sampling times varied from 4-7 $\mathrm{m}$, depending on atmospheric conditions.

\section{Results}

A total number of 114 particle size distributions was measured, from which 17 vertical profiles were constructed, at windspeeds ranging from 2.5 to 13 $\mathrm{m} / \mathrm{s}$. The profiles show the following general features:

(1) both the particle concentrations and their variation with height are strongly dependent on windspeed;

(2) at windspeeds of about $7 \mathrm{~m} / \mathrm{s}$ and higher, maxima are observed in the particle concentrations at about 1-2 m, depending on windspeed;

(3) relative minima are observed at $0.5 \mathrm{~m}$ for the largest particles and at low windspeeds also for the smaller ones; 
(4) at all but the highest winds the concentrations at $11 \mathrm{~m}$ are smaller than at $0.2 \mathrm{~m}$. The high wind result may be due, however, to the remnants of the aerosol produced by gale in the previous days, which was subsiding during the sampling;

(5) above $2 \mathrm{~m}$ the particle concentrations decrease with height;

(6) at a given windspeed, the average concentration gradients decrease with decreasing particle diameter.

The above features are illustrated by the profiles shown in Figs. 2, 3 and 4, measured at low,

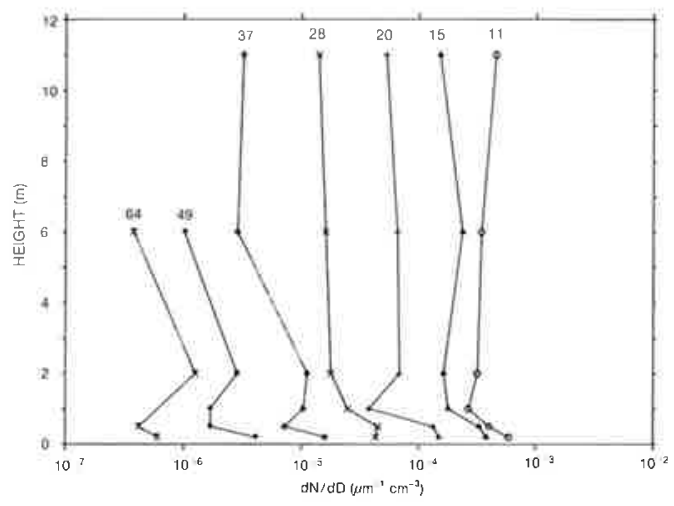

Fig. 2. Vertical profiles of sea-spray particles, measured on June 4 at a windspeed of $5.5 \mathrm{~m} / \mathrm{s}$. RH $=70 \%, H_{\mathrm{s}}=$ $0.5 \mathrm{~m}$, swell $=1.5 \mathrm{~m}$. Parameter is the particle diameter $(\mu \mathrm{m})$ at the indicated ambient relative humidity.

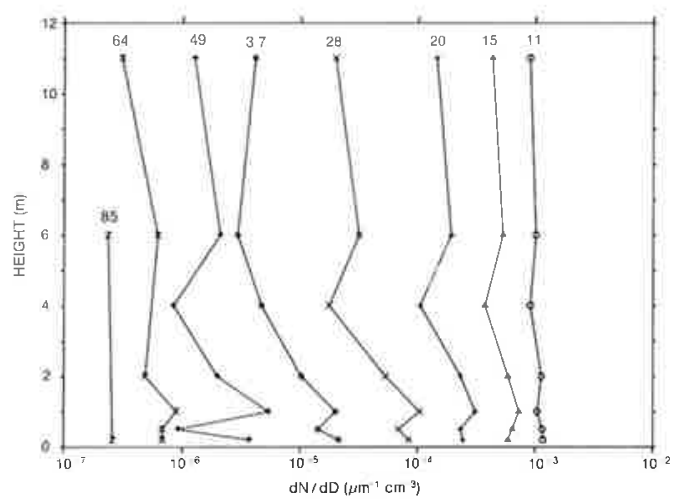

Fig. 3. As Fig. 2, measured on June 22, at a windspeed of $7 \mathrm{~m} / \mathrm{s} . \mathrm{RH}=61 \%, H_{\mathrm{s}}=1.5 \mathrm{~m}$, swell $=2.5 \mathrm{~m}$. Note that for the smallest particles (11 and $15 \mu \mathrm{m}$ ) the minimum at $0.5 \mathrm{~m}$ has vanished. The on-set of a relative maximum at $1 \mathrm{~m}$ is visible for all particles.

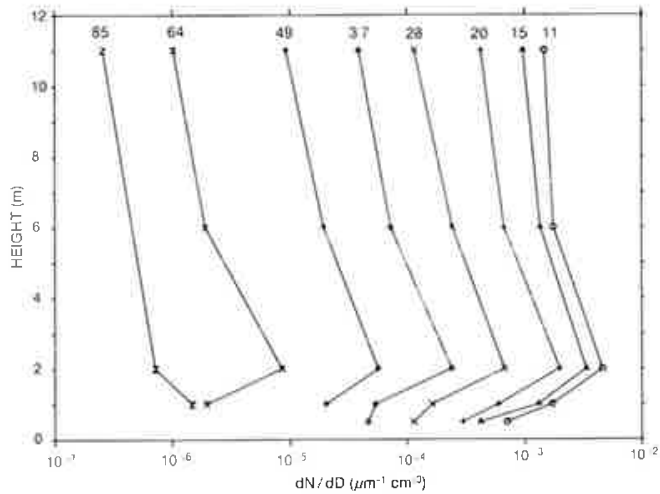

Fig. 4. As Fig. 2, measured on June 13 at a windspeed of $13 \mathrm{~m} / \mathrm{s}$, decreasing after storm during the previous days. $\mathrm{RH}=68 \%, H_{\mathrm{s}}=2.5 \mathrm{~m}$, swell $=3 \mathrm{~m}$. In the rough seas, sampling at $0.2 \mathrm{~m}$ was skipped. Note the very pronounced maximum at $2 \mathrm{~m}$.

moderate and high winds, respectively. For the larger particles the profiles are often not complete over the whole range of heights, due to the combined effects of a limited ejection height, and of the high gravitational forces on these heavy particles, which prevent upward transport by convective and turbulent forces (see also Section 4).

The windspeed dependence of the particle concentrations, as well as the dependence of the particle-concentration/windspeed relationship on particle diameter and on height, are treated in more detail below. All the observed features are explained by the qualitative model outlined in Section 4.

\subsection{Windspeed dependence of particle concentrations}

Concentrations of sea-spray particles are strongly dependent on windspeed (e.g. Monahan et al., 1983; Exton et al., 1985 and references cited therein). Experimental data are often fitted to an exponential relationship of the form:

$\mathrm{d} N / \mathrm{d} D=a \mathrm{e}^{b u}$,

to allow a background component at zero windspeed. Other authors, however, apply a power law fit:

$\mathrm{d} N / \mathrm{d} D=c u^{d}$,

which readily relates the particle concentrations to whitecap coverage (Toba and Chaen, 1973; Mon- 


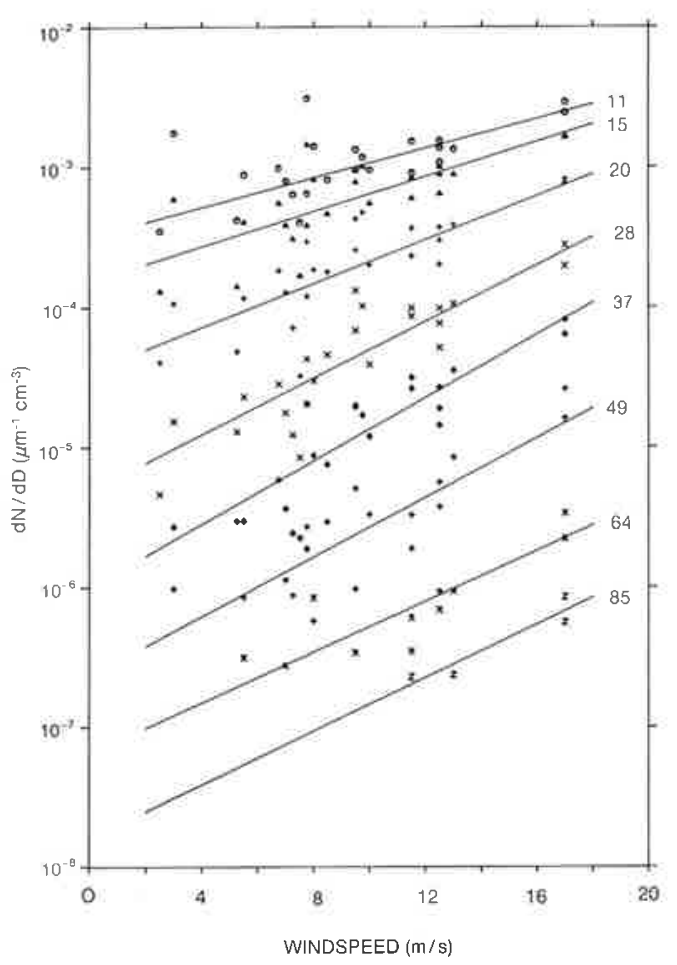

Fig. 5. Scatterplot of $11 \mathrm{~m}$ particle concentrations vs. windspeed. Parameter is the particle diameter $(\mu \mathrm{m})$, corrected to $\mathrm{RH}=80 \%$. Solid lines are least squares fits for each diameter.

ahan et al., 1983a). In eqs. (1) and (2), $\mathrm{d} N / \mathrm{d} D$ $\left(\mu \mathrm{m}^{-1} \mathrm{~cm}^{-3}\right)$ is the particle number concentration $N\left(\mathrm{~cm}^{-3}\right)$ per particle diameter increment $\mathrm{d} D(\mu \mathrm{m})$, and $u$ is windspeed in $\mathrm{m} / \mathrm{s}$ measured at reference height (25 $\mathrm{m}$ at OWS Cumulus); $a, b, c$ and $d$ are constants.
Simple least squares fits were made to eqs. 1 and 2 for particle concentrations at each of the sampling heights from 0.2 to $11 \mathrm{~m}$, and for all particle sizes $(11-85 \mu \mathrm{m})$ for which at least four data points were available. As an example, in Fig. 5 particle concentrations measured at $11 \mathrm{~m}$ are plotted versus windspeed on a semi-logarithmic scale, with particle diameter as parameter. It is noted that the particle concentrations as used in this section have been corrected for relative humidity effects according to the usually applied formulas of Fitzgerald (Fitzgerald, 1975; Fairall et al., 1983):

$\mathrm{d} N / \mathrm{d} D=n^{\prime}(D g(S)) g(S)$.

Here $n^{\prime}$ is the particle size distribution after correction of the particle sizes to humidities of $80 \%$ by (Hughes and Richter, 1980; Fairall et al., 1983):

$D^{\prime}=D g(s)=D(0.81 \exp [0.066 S /(1.058-S)])$,

where $S=0.8$ is the standard saturation ratio.

Least squares fits for each particle size are plotted in Fig. 5 as solid lines. Correlation coefficients were computed after omission of points deviating more than a factor two from the average. In general, these were less than one quarter of the total number of points. Both slope and intercept were hardly affected by this procedure, only the correlation coefficients increased. The results of both the exponential and the power law fits are presented in Tables 1 and 2. The correlation coefficients calculated for the exponential and power law fits are similar, hence a choice between either of these forms should be based on physical

Table 1. Exponents $b$ and correlation coefficients $r$ determined for an exponential fit of particle concentrations to windspeed, for heights $H$ and particle diameters $D$ (eq. 2)

\begin{tabular}{lllllllllllllllll}
\hline$H[\mathrm{~m}]$ & 0.2 & & 0.5 & & 1 & & 2 & & 4 & & 6 & & \multicolumn{2}{c}{11} \\
\hline$D[\mu \mathrm{m}]$ & $b$ & $r$ & $b$ & $r$ & $b$ & $r$ & $b$ & $r$ & $b$ & $r$ & $b$ & $r$ & $b$ & $r$ \\
\hline 11 & 0.16 & 0.81 & 0.11 & 0.52 & 0.085 & 0.54 & 0.19 & 0.88 & 0.16 & 0.86 & 0.046 & 0.38 & 0.12 & 0.88 \\
15 & 0.18 & 0.83 & 0.20 & 0.65 & 0.17 & 0.82 & 0.22 & 0.89 & 0.26 & 0.89 & 0.17 & 0.80 & 0.14 & 0.87 \\
20 & 0.24 & 0.89 & 0.26 & 0.81 & 0.12 & 0.71 & 0.27 & 0.82 & 0.28 & 0.87 & 0.23 & 0.84 & 0.18 & 0.91 \\
28 & 0.31 & 0.89 & 0.15 & 0.78 & 0.17 & 0.59 & 0.34 & 0.84 & 0.30 & 0.81 & 0.30 & 0.87 & 0.23 & 0.94 \\
37 & 0.33 & 0.85 & 0.22 & 0.72 & 0.21 & 0.94 & 0.30 & 0.89 & 0.39 & 0.89 & 0.44 & 0.96 & 0.26 & 0.94 \\
49 & 0.52 & 0.95 & 0.19 & 0.76 & 0.32 & 0.92 & 0.13 & 0.74 & 0.14 & 0.65 & 0.12 & 0.82 & 0.24 & 0.90 \\
64 & 0.53 & 0.40 & 0.20 & 0.50 & 0.066 & 0.35 & - & - & - & - & 0.19 & 0.88 & 0.21 & 0.95 \\
85 & 0.25 & 0.43 & - & - & 0.051 & 0.89 & - & - & - & - & - & - & 0.22 & 0.95 \\
\hline
\end{tabular}


Table 2. Exponents $d$ and correlation coefficients $r$ determined for a power law fit of particle concentrations to windspeed, for heights $H$ and particle diameters $D$ (eq. (2))

\begin{tabular}{lllllllllllllllll}
\hline$H[\mathrm{~m}]$ & 0.2 & & 0.5 & & 1 & & 2 & & 4 & & 6 & & 11 \\
\hline$D[\mu \mathrm{m}]$ & $d$ & $r$ & $d$ & $r$ & $d$ & $r$ & $d$ & $r$ & $d$ & $r$ & $d$ & $r$ & $d$ & $r$ \\
\hline 11 & 0.80 & 0.79 & 0.83 & 0.51 & 0.63 & 0.63 & 1.1 & 0.85 & 0.91 & 0.90 & 0.22 & 0.31 & 1.0 & 0.86 \\
15 & 0.95 & 0.84 & 0.97 & 0.47 & 1.2 & 0.91 & 1.7 & 0.91 & 1.4 & 0.88 & 1.1 & 0.83 & 1.2 & 0.90 \\
20 & 1.3 & 0.89 & 1.1 & 0.60 & 1.5 & 0.92 & 1.9 & 0.91 & 1.5 & 0.91 & 1.4 & 0.88 & 1.4 & 0.90 \\
28 & 1.7 & 0.92 & 1.2 & 0.66 & 1.1 & 0.65 & 2.4 & 0.66 & 1.5 & 0.83 & 2.7 & 0.91 & 2.0 & 0.94 \\
37 & 1.8 & 0.88 & 2.3 & 0.78 & 1.8 & 0.96 & 2.0 & 0.92 & 1.6 & 0.81 & 5.7 & 0.96 & 2.7 & 0.95 \\
49 & 2.8 & 0.98 & 1.2 & 0.76 & 2.2 & 0.89 & 0.63 & 0.61 & 0.61 & 0.55 & 0.60 & 0.72 & 2.2 & 0.81 \\
64 & 3.8 & 0.37 & 1.7 & 0.52 & 0.72 & 0.40 & - & - & - & - & 1.5 & 0.82 & 1.7 & 0.89 \\
85 & 1.9 & 0.42 & - & - & 0.98 & 0.51 & - & - & - & - & - & - & 3.1 & 0.94 \\
\hline
\end{tabular}

arguments. Both are presented here for easy comparison with results from other investigations.

The data in Tables 1 and 2 indicate a dependence of the particle concentration/windspeed relationship on particle diameter as well as on height. In Fig. 6 , the exponents $b$ determined from the exponential fits, eq. (1), are plotted versus particle diameter, with sampling height as parameter. The

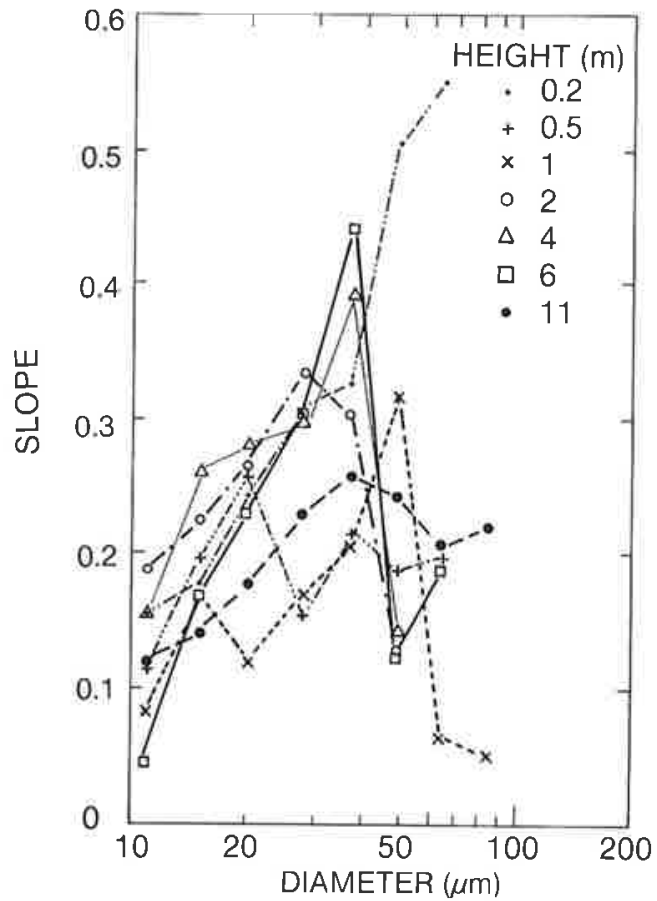

Fig. 6. Exponents for exponential fits of particle concentrations to windspeed, plotted versus particle diameter, for various heights. exponents have a maximum value for particles with diameters around $40-50 \mu \mathrm{m}$. The same conclusion is reached from the power law fit. The height dependence of the particle-concentration/windspeed relationships can be deduced from Fig. 7, where the slopes $b$ determined from exponential fits, eq. (1), are plotted versus sampling height for the various particle diameters. Fig. 7 clearly shows that on the average the influence of windspeed on the particle concentrations is highest at $0.2 \mathrm{~m}$, and between 2 and $6 \mathrm{~m}$, while the influence is relatively low at $1 \mathrm{~m}$. These results are confirmed by our qualitative model, which is discussed below.

For power-law fits the height dependence is less evident than for the exponential fits displayed in Fig. 7. The features are similar, however, though less pronounced.

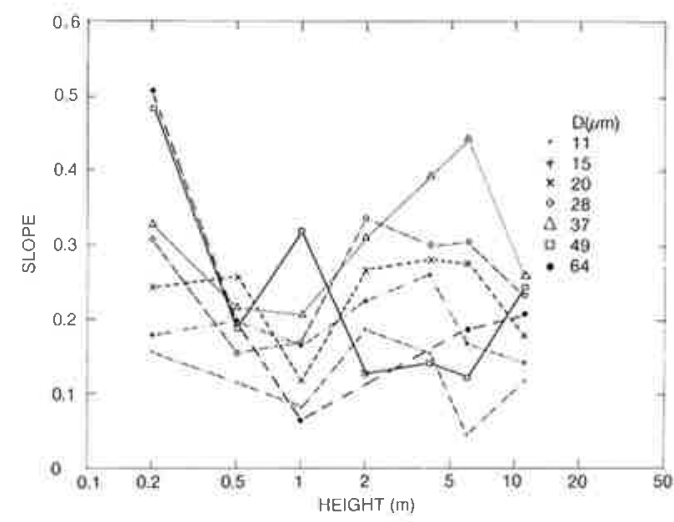

Fig. 7. Exponents for exponential fits of particle concentrations to windspeed, plotted versus height. Parameter is the particle diameter $(\mu \mathrm{m})$. 


\section{Qualitative model}

The results, presented in the previous section, on the vertical profiles of particle concentrations, as well as on the variation in height of the relation between particle concentrations and windspeed are qualitatively explained by the model outlined below. This model is based on previously published observations on particle production and on windwave interaction. The most important of these are summarized and in perspective connected with our observations, before the actual model is explained.

Laboratory studies have shown that droplets ejected into the atmosphere from rising air bubbles reach a maximum height of approximately $20 \mathrm{~cm}$, depending on both bubble size and particle diameter (Blanchard and Woodcock, 1957). Due to gravitational forces the particles fall back unless they are carried up by convection or turbulence. Therefore, in the absence of upward forces particle concentrations are expected to be high in the lower $20 \mathrm{~cm}$ above the instantaneous water level, but with a strong negative vertical gradient. This explains the observed relative minimum at $0.5 \mathrm{~m}$ at low winds. When windspeed increases, this minimum disappears first for the smallest particles, and at higher winds also for the larger particles. This indicates a wind-induced upward air motion close above sea level, which balances gravitational forces and carries the aerosol from the production zone to higher altitudes. Obviously stronger updraught is required as particle size (mass) increases, so according to our observations the upward air motions should be more intense at higher windspeed. Upward air motions have been demonstrated in wind-wave interaction studies (e.g., Koga and Toba, 1981; Koga, 1984; Hsu et al., 1981; Konishi, 1981; Banner and Melville, 1976; Anisimova et al., 1976) and are described by e.g. the theories of Miles and Phillips (cf. Hsu et al. (1981) and references cited therein), which lead to the following qualitative picture. In front of the wave crest, i.e., at the lee, the pressure is slightly reduced, due to the action of the wind blowing over the wave tops. This results in an upward air current. Similarly, at the windward side behind the crest a downward air current is caused by a somewhat higher pressure. As a consequence of the pressure gradient, the air in the wave troughs is slowed down. Thus, the air stream is reversed with respect to the direction of the wavefield as well as with respect to the wind direction above the waves. As a result, in a wave-following coordinate system a circulating air stream exists in and above the wave troughs (see e.g. Fig. 15 in Hsu et al. (1981)). This phenomenon, which obviously is windspeeddependent, has been observed from wind-profile measurements in wave tanks (e.g., Hsu et al. (1981)) and over open water (Anisimova et al., 1976), as well as from flow visualization studies with smoke particles (Konishi, 1981) or bubbles (Banner and Melville, 1976).

The above considerations of aerosol production at the sea surface and the wind structure above the waves lead to a model which qualitatively explains the features observed in the vertical profiles of particle concentrations and their dependence on windspeed. In this model, shown schematically in Fig. 8, the marine atmospheric boundary layer is divided into three parts, which are not strictly separated. The lower part is the production zone with high concentrations of freshly produced sea-spray particles. Here the dynamical processes of production and subsequent gravitational fall-out take place. Since only a small fraction of the particles reach the maximum ejection height (Blanchard and Woodcock, 1957), the particle concentrations in the production zone are expected to have a negative vertical gradient, which explains the observed decrease of the particle concentrations from 0.2 to $0.5 \mathrm{~m}$ at low winds. The upward forces on the particles increase at higher winds due to stronger updraught at the lee of the waves. In high wind conditions these forces act at lower heights as well, since the airstreams close to the air-sea interface will be more intense. This affects lighter (smaller) particles more than heavier

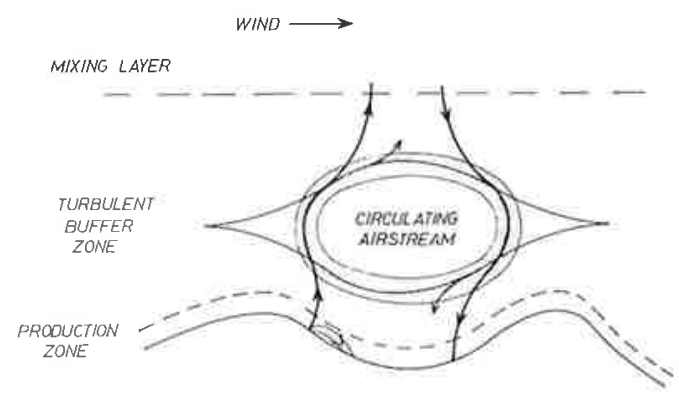

Fig. 8. Schematic representation of the three layer model described in the text (not on scale). 
ones. Consequently, when windspeed increases, the relative minimum at $0.5 \mathrm{~m}$ will first disappear for the smallest particles. At high winds this minimum was only observed for the largest particles under consideration. On the other hand, also the downward forces on the windward side of the waves will increase as windspeed becomes higher, and these downward forces will act closer to the sea surface as well. This will lead to smaller ejection heights but, since we were not able to measure closer than $0.2 \mathrm{~m}$ to the surface, this will lead to an apparent decrease of the concentration gradients. (It is noted that particle concentrations increase with windspeed due to higher production.) In addition particle transport from above increases in high winds, as explained below. Both effects together lead to a change in the near-surface concentration gradients which even might become positive, as observed. Hence both upward transport at the lee of the waves and downward transport at the windward side lead to the observed change in the particle concentrations gradients in the lower $0.5 \mathrm{~m}$.

Due to the updraught, the particles are carried up into the buffer zone, where the concentrations are enhanced due to temporary trapping of the particles in the circulating air stream. Since the intensity of the turbulence depends on windspeed, the particle concentrations are affected by this parameter, as well as their vertical profile. The particles are accelerated by the air stream and, depending on mass (inertia) and position they are forced to follow the trajectory of the air parcels which may lead to temporary trapping of the particles in the buffer zone. This causes the maximum in the concentration profiles at 1-2 m. As a consequence of the growth of the turbulent layer with increasing windspeed (wave height), this maximum also shifts upward, as observed. Particles may leave the buffer layer due to the following processes. The radii of the trajectories followed by the particles will gradually increase due to centrifugal forces. On the other hand, the downward forces, due to the downward air current at the windward side of the crest and due to particle inertia in the airstream at the lower side of the buffer layer, are reinforced by gravitation. Hence it is expected that the majority of the particles will be deposited below the buffer layer into the production zone, and finally back into the water. Due to the enhanced removal rates in increasing winds the increase of particle concentrations in the buffer layer is less than expected from the increase in production and upward transport. Since the downward forces causing removal have more effect in the lower part of the circulating airstream, the maximum in the concentration profile is expected to shift upward. Therefore the height at which this maximum occurs depends on windspeed both due to the growth of the buffer layer and due to the increase in turbulence intensity.

Gravitation not only reinforces the downward force of the turbulence, at the top side it tends to pull the particles deeper into the circulating air stream. Yet it is obvious that a fraction of the particles is transported from the buffer layer up into the mixing layer. Most probably this will occur at the lee of the crests, where the updraught is most intense, while freshly produced particles are caught still having a relatively high vertical velocity component left after ejection. However, particles transported out of the buffer layer by inertia must be taken into account as well, since these can be carried up by convection in the separating flow.

In the mixing layer the concentrations will obviously be smaller than in the buffer zone. Vertical transport in the mixing layer takes place by diffusive, turbulent and gravitational forces. Aerosol concentrations are dependent on stability, entrainment, subsidence etc., as described, e.g. in Fairall et al. (1984), as well as on air-mass history. The latter effect is illustrated by the relatively high concentrations observed at $11 \mathrm{~m}$ in a (decreasing) wind of $13 \mathrm{~m} / \mathrm{s}$, which are suspected to be the remainder of gale during the previous days. Due to the short residence times of the giant particles considered here, such high concentrations are not common.

The observed windspeed dependence of the particle concentrations can also be explained by this model. The production of particles by the rising bubble mechanism is enhanced by entrainment of air by wind-induced wave breaking. Therefore, in the production zone the particle concentrations will strongly increase with windspeed. However, particle removal increases with windspeed as well. Consequently, the exponents in the particle-concentrations/windspeed relationships are smaller in the mixing layer than in the production zone. In the buffer layer the number of trapped particles increases with windspeed due to more intensive air circulations. This is confirmed by the data in Tables 
1 and 2, and particularly by Fig. 7. Fig. 7 also clearly displays a minimum windspeed dependence of particle concentrations at approx. $1 \mathrm{~m}$, i.e. in the buffer zone. This might have two causes. As discussed above, at higher windspeeds, and the accompanying higher waves, the depth of the buffer zone increases, leading to a dilution of the particle concentrations. Since the two effects of enhanced production and simultaneously stronger dilution have an opposite effect, the particle concentrations at $1 \mathrm{~m}$ will be less dependent on windspeed than at other heights. A further indication for this explanation is the relatively high windspeed dependence of the concentrations at $2 \mathrm{~m}$. The second reason for a minimum in the windspeed dependence at $1 \mathrm{~m}$ is removal which will have a strong effect on the particle concentrations at the lower side of the circulating airstream, as explained.

As indicated above, the concentrations of the largest particles are more affected by windspeed than the smaller ones. This too, is reflected by the windspeed dependence of the particle concentrations, as was shown in Fig. 6. Apparently for particles with diameters around $40-50 \mu \mathrm{m}$, which display the highest dependence on windspeed, gravitational forces are most effectively balanced by the upward turbulent forces.

\section{Summary and discussion}

Particle size distributions in the 10-100 $\mu \mathrm{m}$ diameter range were measured at heights of 0.2 , $0.5,1,2,4,6$ and $11 \mathrm{~m}$ above sea level with a Rotorod inertial impactor. As far as we know, these aerosol profiles are the first ones ever measured close above sea level on the high seas. Both the particle concentrations and their vertical distributions appear to be strongly dependent on windspeed. Above $2 \mathrm{~m}$ the concentrations have a negative gradient, in agreement with earlier studies (Chaen, 1973; Preobrazhenskii, 1973). The most important features of the profiles are the maxima observed at winds of about $7 \mathrm{~m} / \mathrm{s}$ and higher at heights of 1-2 $\mathrm{m}$ above sea level, and the relative minimum at $0.5 \mathrm{~m}$ at low winds. For the smaller particles this minimum disappears when windspeed increases, although for the largest droplets $(>49 \mu \mathrm{m})$ it is still observed at the highest wind ( 10 $\mathrm{m} / \mathrm{s}$ ) in which particle profiles were measured from $0.2 \mathrm{~m}$. A strong dependence of particle con- centrations on windspeed is expected because of entrainment of air by wind-induced wave breaking, which increases the bubble population and thus gives rise to enhanced production of film and jet drops by the rising bubble mechanism (Blanchard and Woodcock, 1957; Blanchard, 1983; Monahan et al., 1983b). At higher winds $(>9 \mathrm{~m} / \mathrm{s})$ wave disruption gains importance (Monahan et al., 1983b). Also vertical transport is likely to be facilitated in high winds. However, a certain time lag is expected between the increase of windspeed and its effect on aerosol loading. Experimental evidence for time lags on the order of several hours were reported, although other factors may have been of influence on these results as well (Exton et al., 1985). Our data were fitted to instantaneous windspeed at $25 \mathrm{~m}$, averaged over $10 \mathrm{~min}$. Both power law and exponential particle-concentration/ windspeed relationships were deduced from a statistical approach, for particles over the whole range of diameters $(11-85 \mu \mathrm{m})$ and at all heights $(0.2-11 \mathrm{~m})$. Both the exponential and power law relations fit equally well and any preference should be based on physical arguments. The exponential form is commonly used to describe the salt-mass loading of the atmosphere, since it allows a background concentration at zero windspeed. Power law fits are made because this form applies to whitecapping/windspeed relations (Toba and Chaen, 1973; Monahan et al., 1983a). Hence a power law relationship might be more fundamental because it relates the aerosol concentration to the production mechanism. For both cases the values of the exponents appear to be a function of both particle diameter and height. The influence of windspeed is highest for particles of about $40 \mu \mathrm{m}$. Particle concentrations are most affected by windspeed at heights of $0.2 \mathrm{~m}$ and between 2 and $6 \mathrm{~m}$, while at $1 \mathrm{~m}$ the influence of windspeed is relatively low. Extensive comparisons of reported particleconcentration/windspeed relationships were made by Exton et al. (1985).

All of the observed features in the aerosol profiles, as well as the windspeed dependence of the particle concentrations at various heights, are explained by a qualitative model based on literature data from wind-wave interaction studies as well as from laboratory studies on production of sea-spray particles by the rising bubble mechanism. In this model the lower part of the marine atmospheric boundary layer is divided into three parts: 
- the production zone adjacent to the instantaneous air/sea boundary;

- the buffer zone, i.e., the turbulent layer where particle concentrations are enhanced due to temporarily trapping of the particles in a circulating air stream;

- the mixing layer, where particle concentrations are determined by vertical transport by gravitational and convective forces, as well as by air mass history; the vertical structure depends on stability, entrainment, etc., as described e.g. in Fairall et al. (1984).

The depth of the three layers depends on windspeed. The transition between them is gradual.

In this model, humidity effects were not taken into account. Freshly produced particles having the composition of sea water, will evaporate until they are in equilibrium with their surroundings. For the large particles under consideration it will take some time to reach this equilibrium (Fairall, 1984). Therefore it would be useful to measure relative humidity profiles simultaneously with the particle spectra.

Some of the features in the vertical profiles measured on the high seas are similar to the results by Koga and Toba (1981) from laboratory studies, viz., the maximum near wave height and the reduction of the particle concentrations above the wave tops with respect to those in the troughs. These results apply to particles in the sub-mm range. The range of windspeeds in this laboratory experiment was too limited to study the influence of this parameter. This is the only other experiment we know of, where particle concentrations were determined in wave troughs.

\section{Conclusion}

The profiles of particle size distributions measured in the lower $11 \mathrm{~m}$ above the sea surface display a number of features which are all explained by a qualitative model. In the near future, we hope to develop a model that quantitatively describes the observed aerosol profiles. Together with mixing layer models developed by others (e.g., Fairall et al. (1984)) this might result in quantitative predictions of aerosol behaviour in various meteorological conditions, which is of importance for a wide range of applications such as optical and meteorological modelling of the atmosphere and environmental studies.

\section{Acknowledgements}

The measurements aboard MS Cumulus were supported by a grant from the NATO Special Fund for Cooperative Planning. C. W. Lamberts, L. H. Cohen, M. M. Moerman and P. D. Piket are acknowledged for their continuous stimulations and practical assistance.

\section{REFERENCES}

Anisimova, Ye. P., Speranskaya, A. A. and Likhacheva, O. N. 1976. Deformation of the wind velocity profile over developing wind waves. Izv., Atmos. Oceanic Phys. 12, 748-754.

Banner, M. L. and Melville, W. K. 1976. On the separation of air flow over water waves. J. Fluid Mech. 77, 825-842.

Blanchard, D. C. 1983. The production, distribution, and bacterial enrichment of the sea-salt aerosol. In: Air-sea exchange of gases and particles (eds. P. S. Liss and W. G. N. Slinn). Dordrecht/Holland: D. Reidel Publ. Comp., 407-454.

Blanchard, D. C. and Woodcock, A. H. 1957. Bubble formation and modification in the sea and its meteorological significance. Tellus 9, 145-158.

Blanchard, D. C. and Woodcock, A. H., 1980. The production, concentration, and vertical distribution of the sea-salt aerosol. Ann. NY Acad. Sci. 338 330-347.

Blanchard, D. C., Woodcock, A. H. and Cipriano, R. J. 1984. The vertical distribution of the concentration of sea salt in the marine atmosphere near Hawaii. Tellus $36 B, 118-125$.

Chaen, M. 1973. Studies on the production of sea-salt particles on the sea surface. Mem. Fac. Fish., Kagoshima Univ. 22, 49-107.

Emonds, R. L. 1972. Collection efficiency of rotorod sampler for sampling fungus spores in the atmosphere. Plant Dis. Rep. 56, 704-708.

Exton, H. J., Latham, J., Park, P. M., Perry, S. J., Pounder, C., Smith, M. H. and Allan, R. R. 1985. The production and dispersal of marine aerosol. $Q . J . R$. Meteorol. Soc. 111, 817-837.

Fairall, C. W. 1984. Interpretation of eddy-correlation 
measurements of particulate deposition and acrosol flux. Atmos. Environ. 18, 1329-1337.

Fairall, C. W., Davidson, K. L. and Schacher, G. E. 1983. An analysis of the surface production of sea-salt aerosols. Tellus 35B, 31-39.

Fairall, C. W., Davidson, K. L. and Schacher, G. E. 1984. Application of a mixed-layer model to aerosols in the marine boundary layer. Tellus 36B, 203-211.

Fairall, C. W. and Larsen, S. E. 1984. Dry deposition, surface production and dynamics of aerosols in the marine boundary layer. Atmos. Environ. 18, 69-77.

Fitzgerald, J. W. 1975. Approximation formulas for the equilibrium size of an aerosol particle as a function of its dry size and composition and the ambient relative humidity. J. Appl. Meteorol. 14, 1044-1049.

Hughes, H. G. and Richter, J. H. 1980. Extinction coefficients calculated from aerosol size distributions measured in the marine environment. Opt. Engin. 19, $616-620$.

Hsu, C.-T., Hsu, E. Y. and Street, R. L. 1981. On the structure of turbulent flow over a progressive water wave: theory and experiment in a transformed, wave-following co-ordinate system. J. Fluid Mech. 105, 87-117.

Koga, M. 1984. Dispersal of droplets over breaking wind waves under the direct action of wind. J. Oceanogr. Soc. Japan 40, 29-38.

Koga, M. and Toba, Y. 1981. Droplet distribution and dispersion processes on breaking wind waves. Sci. Rep. Tohoku Univ., ser. 5. (Tohoku Geophys. J.) 28, $1-25$.

Konishi, T. 1981. Analysis of wind structure over water waves using a flow visualization method. Pap. Meteorol. Geophys. 32, 99-108.

de Leeuw, G. 1986. Size distributions of giant aerosol particles close above sea level. J. Aerosol Sci. 17, in press.

Monahan, E. C. 1968. Seaspray as a function of low elevation windspeed. J. Geophys. Res. 73, 1127-1137.
Monahan, E. C. 1985. The ocean as a source for atmospheric particles. In: Proc. Vol. of NATO Adv. Study Inst. The rôle of air-sea exchange in geochemical cycling, Bombannes (France), 16-27 September. D. Reidel Publ. Comp., to be published.

Monahan, E. C., Fairall, C. W., Davidson, K. L. and Boyle, P. J. 1983a. Observed inter-relations between $10 \mathrm{~m}$ winds, ocean whitecaps and marine aerosols. $Q$. J. R. Meteorol. Soc. 109, 379-392.

Monahan, E. C., Spiel, D. E. and Davidson, K. L. 1983 b. Model of marine aerosol generation via whitecaps and wave disruption. Ninth Conf. on Aerospace and Aeronautical Meteor, June 6-9, Omaha, Nebr.

Preobrazhenskii, L. Yu. 1973. Estimate of the content of spray-drops in the near-water layer of the atmosphere. Fluid Mech.-Soviet Res. 2, 95-100.

Slinn, W. G. N. 1983. Air-to-sea transfer of particles. In: Air-sea exchange of gases and particles (eds. P. S. Liss and W. G. N. Slinn). Dordrecht/Holland: D. Reidel Publ. Comp., 299-405.

Slinn, S. A. and Slinn, W. G. N. 1980. Predictions for particle deposition on natural waters. Atmos. Environ. 14, 1013-1016.

Toba, Y, 1965. On the giant sea-salt particles in the atmosphere II. Theory of the vertical distribution in the 10-m layer over the ocean. Tellus 17, 365-382.

Toba, Y. and Chaen, M. 1973. Quantitative expression of the breaking of wind waves on the sea surface. Rec. Oceanogr. Works Japan 12, I-11.

Van de Ven, M. J. M., Noorman, C. J. and Lamberts, C. W. 1980. Coastal aerosol measurements. J. Aerosol Sci. 11, 281-292.

Vrins, E. and Hofschreuder, P. 1983. Sampling total suspended particulate matter. J. Aerosol Sci. 14, 318-322.

$\mathrm{Wu}$, J. 1979. Spray in the atmospheric surface layer: review and analysis of laboratory and oceanic results. J. Geophys. Res. 84, 1693-1704. 
